\title{
Blasting Vibration Control Using an Improved Artificial Neural Network in the Ashele Copper Mine
}

\author{
Shida Xu $(\mathbb{D}$, Tianxiao Chen $(\mathbb{D}$, Jiaqi Liu $(\mathbb{D}$, Chenrui Zhang $(\mathbb{D}$, and Zhiyang Chen $(\mathbb{D}$ \\ Key Laboratory of Ministry of Education on Safe Mining of Deep Metal Mines, Northeastern University, Shenyang, \\ Liaoning 110819, China \\ Correspondence should be addressed to Shida Xu; xushida@mail.neu.edu.cn
}

Received 5 March 2021; Revised 17 April 2021; Accepted 29 May 2021; Published 11 June 2021

Academic Editor: Qi ZHAO

Copyright (c) 2021 Shida Xu et al. This is an open access article distributed under the Creative Commons Attribution License, which permits unrestricted use, distribution, and reproduction in any medium, provided the original work is properly cited.

Blasting is currently the most important method for rock fragmentation in metal mines. However, blast-induced ground vibration causes many negative effects, including great damage to surrounding rock masses and projects and even casualties in severe cases. Therefore, prediction of the peak particle velocity (PPV) caused by blasting plays an important role in reducing safety threats. In this paper, a genetic algorithm (GA) and an artificial neural network (ANN) algorithm were jointly used to construct a neural network model with a 4-5-1 topology to predict the PPV. For this model, the ANN parameters were optimized using the GA, and the deviating direction, horizontal distance, vertical distance, Euclidean distance, explosive type, burden, hole spacing, and maximum charge per delay were used as input information. Moreover, principal component analysis (PCA) was used to extract the first four principal components from the eight input factors as the four inputs of the ANN model. The model was successfully applied to protect an underground crushing cave from blasting vibration damage by adjusting the blasting parameters. Compared with several widely used empirical equations, the GA-ANN PPV prediction model produced significantly better results, while the Ambraseys-Hedron method was the best of the empirical methods. Therefore, the improved GA-ANN model can be used to predict the PPV on site and provide a reference for the control of blasting vibration in field production.

\section{Introduction}

Blasting is currently the most economical and widely used method for artificial caving in underground mines. However, the utilization efficiency of the energy released by explosive charges is very low. According to statistics, only $20-30 \%$ of the explosive energy is used for rock fragmentation, and the rest of the energy is lost in other forms, resulting in some negative effects, such as surrounding rock vibration, flyrock, shock waves, tremendous noise, and a large amount of released heat [1-4]. The negative effects result in a large amount of energy loss and cause severe damage to the surrounding rock masses, which easily leads to rock bursts, large-scale collapses, and other disasters, further threatening the safety of underground personnel and equipment [5-8]. In particular, as the mining depth increases, the geostatic stress also gradually increases, and the issue of blast-induced rock bursts becomes increasingly prominent. According to statistics, approximately twothirds of rock bursts in mines occur as a result of blastinduced disturbances [9]. The roadway of the $-647 \mathrm{~m}$ middle section of the Hongtoushan copper mine experienced severe damage over a distance of tens of meters. According to the literature, the peak particle velocity (PPV) is the most commonly used predictor to evaluate blasting vibration [10]. Therefore, conducting studies on PPV prediction would help control the blasting vibration and reduce the corresponding hazards.

According to the literature, the PPV is influenced by many factors, including the maximum charge per delay, burden, hole spacing, total charge, number of delays, rock mass quality, and stress $[11,12]$. Many scholars have studied the relationship between these influencing factors and the PPV. Based on these relationships, empirical methods, numerical simulation methods, linear regression methods, and other methods have been used to predict the PPV, and 
many meaningful achievements have been obtained [4]. To date, there is still no widely accepted method. In recent decades, with the development of computer technology, neural networks have been increasingly applied in PPV prediction due to the advantages of self-adaptivity and nonlinearity $[13,14]$.

In a study on the blasting parameter-based prediction of blasting vibration using a neural network approach, Singh and Singh [15] found that the neural network approach accurately predicted ground vibration, and the error did not increase as the number of inputs and nonlinearity increased. Khandelwal and Singh [16] used an artificial neural network (ANN) approach to predict the blast-induced ground vibration of a surface coal mine and compared it with the conventional statistical analysis method. Khandelwal and Singh [17] established an ANN model to predict blast-induced ground vibration. The model contained 10 input parameters, 15 hidden neurons, and two output parameters. A comparative analysis demonstrated that the model has a higher prediction accuracy than conventional predictors and multivariate regression analysis. Monjezi et al. [18] established a four-layer neural network model with a 4-1085-1 topology to predict the PPV and determined the distance from the blasting face to be the parameter that had the greatest impact on the PPV by sensitivity analysis. Amnieh et al. [19] used an ANN to establish the relationship between the PPV, block volume, explosive types, and blasting parameters and used this relationship to determine the appropriate burden, hole spacing, and maximum charge corresponding to the PPV values for a safe blasting pattern. Álvarez-Vigil et al. [20] divided the influencing factors into two categories, viz., environmental conditions and waveform parameters, proposed an ANN model for the prediction of ground vibration caused by blasting in an open-pit mine, and verified the effectiveness of this method by comparing it with multiple linear regression. Jayawardana et al. [21] used a neural network to study the relationship between the vibration mitigation effects of dual in-filled trenches under different conditions and the control parameters and established a vibration prediction model based on the relationship.

Some scholars combined the neural network method with other methods for the prediction of blasting vibration. Verma and Singh [22] established a PPV predictor using a genetic algorithm (GA) based on 127 data sets. They compared its predictors with traditional vibration predictors, multivariate regression analysis, and ANNs and discussed the superiority of the GA network. Mohamed [23] separately used an ANN and fuzzy logic analysis to predict the PPV and found that the fuzzy model had a slightly higher prediction accuracy than the ANN model. Armaghani et al. [24] proposed a flyrock and PPV prediction model based on the particle swarm optimization (PSO) algorithm and an ANN and input the top 10 influential blasting parameters to estimate the flyrock and PPV. They found that the maximum charge per delay was the blasting parameter that influenced the flyrock distance and PPV the most. Hajihassani et al. [25] used a hybrid ANN and PSO algorithm to predict the air blast overpressure and obtained a training correlation coefficient of 0.94 , indicating that the PSO-based ANN model has a high prediction accuracy. Hajihassani et al. [26] established a hybrid algorithm model for PPV prediction (an ANN algorithm and an imperialist competitive algorithm (ICA)), the ICA-ANN model, based on the blasting data of a granite quarry in Malaya, and the application results showed that the ICA-ANN model has a high prediction accuracy. Azimi et al. [27] used the monitoring data of blast-induced ground vibration from the Sungun copper mine to establish a GA-ANN prediction model and found that the prediction accuracy was higher when the horizontal and radial distances were replaced with the modified radial distance. Nguyen et al. [28] analyzed and affirmed the prediction accuracy of blast-induced PPV using a hybrid model based on the hierarchical $k$-means (HKM) clustering algorithm and an ANN (an HKM-ANN model) and a hybrid model based on fuzzy $c$-means clustering (FCM) and support vector regression (SVR) and concluded that the HKM algorithm could effectively improve the prediction accuracy of ANN models.

Based on the monitoring data of ground vibration caused by underground blasting in the Ashele copper mine, the present study established a PPV prediction model by combining the GA with the ANN. In this model, the ANN parameters were optimized by the GA, and the optimal number of nodes and connection weights of the hidden layers in the neural network were obtained. The model was applied to provide guidance for optimizing the blasting parameters and controlling blasting vibration.

\section{Combined Method Based on a Neural Network and GA}

The backpropagation (BP) network is currently one of the most widely used neural network models. The BP network generally consists of an input layer, hidden layers, and an output layer. The learning algorithm of the BP network can be summarized as the process of iteratively performing forward computation, reverse computation, and weight and threshold updating according to a given sample set. The training weight coefficient is reversed. The error of the middle layer is obtained based on the error of the output, and then the weight coefficient of the input is obtained. This process is repeated until the correct output is obtained. The $\mathrm{BP}$ network is essentially the input-output mapping and does not require any precise mathematical expression between the input and the output. As long as a known model is used to train the BP network, the network has the capability of mapping between input and output pairs. It actually transforms the input/output problems of a sample set into a nonlinear optimization problem and uses the most common gradient descent optimization method and iterative operation to solve for the weights.

The neural network uses a gradient descent-based BP algorithm and its variants, and its dependence on the initial weight selection inevitably leads to the problem of a local optimum, resulting in a decrease in the reliability of the neural network approach. In view of the shortcomings of the BP neural network, this paper adopts an improved GA-BP 
neural network to overcome the shortcomings of the BP neural network. The GA simulates the evolutionary phenomena of biological inheritance, genetic variation to adapt to the environment, and survival of the fittest in nature [29]. It treats each solution as a chromosome, each element of the solution as a gene, and all chromosomes as a population. The chromosomes are evaluated using a predetermined objective function to calculate the fitness. The algorithm initially randomly generates some chromosomes, performs genetic operations such as genetic inheritance and variation on the chromosomes based on the fitness, selects chromosomes with a high fitness, and conducts the next generation of genetic operations to obtain new populations. Because each iteration selects the elites from the population of the previous generation, the new generation inherits the superior genes of the previous generation and reorganizes in time to produce better chromosomes and eliminate defective new individuals. Therefore, through repeated iterations, the solution evolves toward the optimal solution until the termination condition is met. Due to the high level of parallelism, the GA has been widely applied in neural networks.

\section{Background of the Ashele Copper Mine}

3.1. Production and Monitoring of the Ashele Copper Mine. The Ashele copper mine, located in Xinjiang Province in China, has a copper reserve of 920,000 tons, with an average grade of $2.4 \%$. The main ore body has a strike length of $700 \mathrm{~m}$, an average thickness of $20 \mathrm{~m}$ to $45 \mathrm{~m}$, and an inclination of $55^{\circ}$ to $85^{\circ}$. The deposit is mainly formed by volcanic ejection and deposition and is then remolded by hydrotherm fluids. The average rock quality designation (RQD) of the surrounding rock in the hanging wall is $81.2 \%$, with $41.5 \%$ of the surrounding rock in the footwall and $70.9 \%$ of the ore body. The current mining depth exceeds $1000 \mathrm{~m}$, with $1243 \mathrm{~m}$ of the shaft length. The hanging wall of the ore body is mainly composed of dacite porphyry, and the footwall of the ore body is mainly basalt, with chalcopyrite as the main mineral. The results of an in situ stress test showed that the maximum horizontal principal stress at the maximum mining depth was close to $45 \mathrm{MPa}$, with an inclination of $18^{\circ}$, which was almost perpendicular to the strike of the ore body, and the minimum principal stress was approximately $20 \mathrm{MPa}$, with an inclination of $64^{\circ}$. The Ashele copper mine mainly employs the large-diameter deep-hole mining method, which allows a high production capacity. Ores were gathered from the bottom using trenches. After the formation of the trenches, the vertical crater retreat (VCR) method was adopted to form cutting grooves at the mining face, and then large-hole lateral caving was used for mining. The Ashele copper mine produces 6,000 tons of ore daily; the charge for a single blast is as high as 4 tons, and the maximum charge per delay reaches $400 \mathrm{~kg}$. Frequent blast and stress transfers due to excavation disturb the surrounding rock mass of roadways, in some cases causing severe damage and requiring significant effort to maintain and rehabilitate the secondary support. Stope collapses have occurred at two different levels of the Ashele copper mine, which were attributed to the blasting disturbance of adjacent stopes after microseismic activity analysis [30].

The NUBOX-6016 intelligent vibration monitor and the NUBOX-6016 portable data acquisition device used in this study were designed based on optimization tests for blasting, vibration, shock, and noise on site (see Figure 1). The instruments can digitally convert and store the dynamic and static analog signals generated by sensors (including the acceleration, pressure, strain, and temperature) and have a triggering mechanism to ensure the correct recording of signals only for the features concerned. They allow data acquisition for up to 2048 segments to achieve continuous automatic recording of multisegment vibration signals. The maximum sampling frequency is $200 \mathrm{kHz}$. The signal-tonoise ratio (SNR) is $\geq 62 \mathrm{~dB}$. The minimum sampling frequency is $5 \mathrm{~Hz}$. This equipment is equipped with a TP3V-4.5 sensor, which has the advantages of easy installation, high robustness, reliability, compactness, high measurement precision, and strong anti-interference. The stope of the Ashele copper mine adopted retreats mining from the hanging wall to the footwall, and during the blasting vibration field test, the monitoring points were placed in the footwall of the ore body to avoid measuring the blasting vibration waves that propagated through the goaf.

Every two or three levels are used as a mining combination in the Ashele copper mine. The mining sequence is from bottom to top in the mining combination. At present, the first stope at a depth of $1012 \mathrm{~m}$ is to be mined according to the production plan. Unfortunately, a crushing chamber is located near the stope, which is used to crush the ore produced below $600 \mathrm{~m}$ depth. Apparently, it has a long service cycle. Therefore, it is necessary to control the PPV of the stope blast to protect the crushing chamber.

3.2. Factors Controlling the $P P V$. Samples of the inputs and outputs should be offered to train and test the blasting vibration prediction model based on the neural network. The related factors concerning the blasting vibration should be selected carefully. Then, the inputs of the ANN model, including blasting parameters and field conditions, were determined after investigation. Field conditions mainly include the buried depth, maximum principal stress, deviating direction, horizontal distance, vertical distance, and Euclidean distance. The blasting parameter mainly contains the explosive type, burden, hole spacing, delay number, total charge, and maximum charge per delay.

\subsubsection{Weak Effect of Burial Depth and Maximum Stress.} There are few studies considering the impact of the buried depth and the maximum stress on blasting vibration. Little correlation between the buried depth, the maximum stress, and the PPV was found before. In this paper, the blasting vibration at a deeper level was studied based on the shallow level, so the two impactors were taken to analyze the impact on the PPV. The blasting vibration data were all collected at $562 \mathrm{~m}$ depth, $762 \mathrm{~m}$ depth, and $912 \mathrm{~m}$ depth, which were the main mining levels in the Ashele copper mine. The PPV values of different depths and maximum principal stresses 


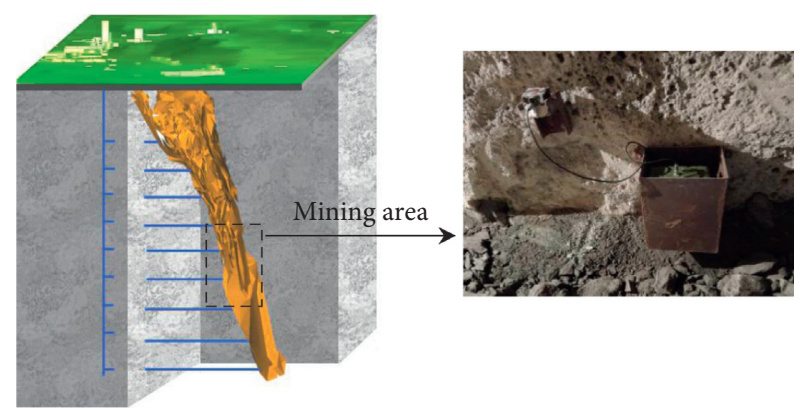

FIgURE 1: Field test of blasting vibration in mining area.

are shown in Figure 2. The PPV tends to increase with increasing burial depth. It can be predicted that the influence of burial depth on PPV will become increasingly prominent with increasing mining depth. According to the results of the in situ stress test of the Ashele copper mine, the maximum principal stress of the in situ stress is near horizontal and approximately perpendicular to the ore body. In addition, there is little change in the direction of the maximum principal stress. Hence, only the value of maximum principal stress is considered for the model. The PPV grows with increasing maximum horizontal principal stress. However, the correlation coefficients between the buried depth, maximum stress, and PPV are small. It can be concluded that there is no significant correlation between them. Therefore, the buried depth and the maximum stress are not taken as the inputs of the PPV prediction. At the same time, the correlation coefficients between the delay number, total charge, and PPV are small, illustrating that the two factors have little impact on the PPV. Therefore, the delay number and the maximum stress are eliminated.

3.2.2. Deviating Direction. The scatterplot matrix of the PPV database with correlation is shown in Figure 3. The deviating direction is the angle between the stope centerline and the line linking the blasting center and the monitoring point. The deviating direction is a positive number when the stope centerline rotates clockwise. Otherwise, the deviating direction is a negative number. Apparently, the PPV is the smallest when the deviating direction is 0 . With the increase in the absolute value of the deviating direction, the PPV rises gradually. The correlation coefficient between the deviating direction and PPV is 0.307 , and a significance test finds significance at the level of 0.05 . It can be concluded that the deviating direction has some influence on the blasting vibration.

\subsubsection{Horizontal Distance, Vertical Distance, and Euclidean} Distance between the Blasting Source and Monitoring Point. Most empirical formulas incorporate the influence of propagation distance on blasting vibration. The horizontal distance is the horizontal projection of the distance between the blasting center and the monitoring point. Clearly, there is a high correlation between the horizontal distance and PPV. As we thought, the PPV decreases as the horizontal distance increases. Similar to the horizontal distance, the

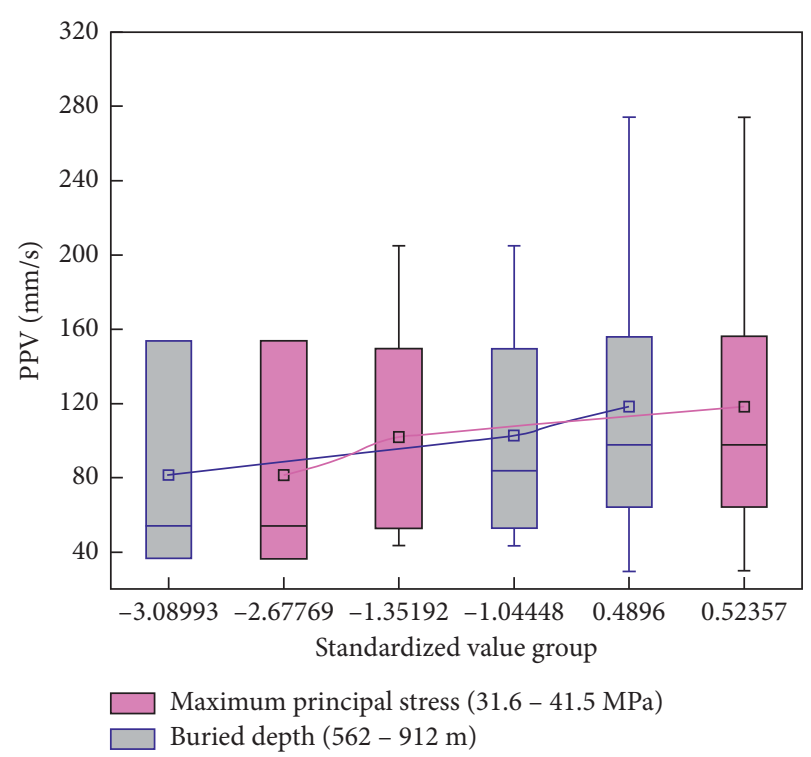

FIgURE 2: Range of the PPV with different burial depths and the maximum principal stress.

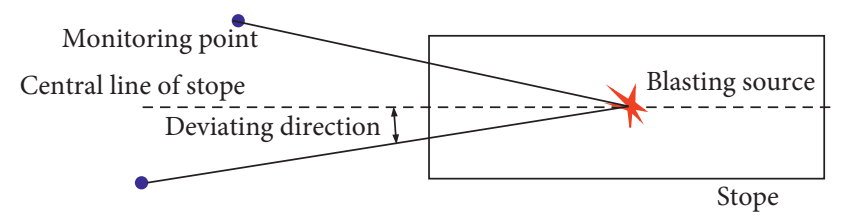

FIgURE 3: Deviating direction schematic diagram.

vertical distance, which is the vertical projection of the distance between the blasting center and the monitoring point, has an obvious influence on blasting vibration. However, there are some differences between the influences of horizontal distance and vertical distance on blasting vibration. The vertical propagation distance should be considered when studying the propagation of blasting vibration at different levels. With the increase in the Euclidean distance between the blasting center and monitoring point, the PPV decays gradually. In other words, with increasing horizontal distance, vertical distance, and Euclidean distance, the PPV decreases gradually. The correlation coefficients of the horizontal distance, vertical distance, and Euclidean distance are $0.607,-0.587$, and -0.73 , respectively. They are significantly correlated with the PPV at the $99 \%$ confidence level. Therefore, they are all incorporated into the PPV prediction model.

3.2.4. Factor of Explosive Type. The blasting speed, power and other parameters vary greatly for different explosive types. In this paper, two types of explosives are mainly used to break the rock. They are emulsion explosive and ammonium oil explosive, which are represented by 1 and 2, respectively. The blasting vibration generated by blasting with emulsion explosive is significantly greater than that of blasting with ammonium oil explosive. The correlation coefficient between the explosive type and PPV is -0.392 , 
which is significant at the 0.01 level. According to the theory proposed by Cohen [31], an absolute value of coefficient greater than 0.3 can be regarded as moderate correlation. Consequently, the explosive type is taken as one of the inputs of the model.

3.2.5. Burden. The burden is the distance from the explosive cartridges to the free surface. When the burden is less than $2.5 \mathrm{~m}$, the PPV increases with decreasing burden. Moreover, the PPV increases with the growth of the burden when the burden is more than $2.5 \mathrm{~m}$. The correlation coefficient between the burden and PPV is 0.324 , which is relatively small. However, a significance test identifies significance at the level of 0.05 . Therefore, the burden can be considered to have an impact on the PPV and can be used to predict PPV.

3.2.6. Hole Spacing. With increasing hole spacing, the mean values and the minimum values of the PPV present an increasing trend. When the hole spacing is $2.0 \mathrm{~m}$, the PPV values were relatively concentrated. The value range of the PPV is large at most hole spacings. The correlation coefficient between the hole spacing and PPV is 0.350 , which is significant at the 0.05 level. As a result, the hole spacing is selected as the input of the model.

3.2.7. Maximum Charge per Delay. Similar to the distance, the maximum charge per delay often appears in empirical formulas. When the maximum charge per delay equals $220 \mathrm{~kg}$, the PPV is the smallest according to the fitting curve. The PPV increases gradually with the increase in the maximum charge per delay after exceeding $220 \mathrm{~kg}$. The PPV also increases with the decrease in the maximum charge per delay when it is less than $220 \mathrm{~kg}$. The correlation coefficient between the maximum charge per delay is 0.381 , which is significant at the level of 0.01 (see Figure 4). Hence, the maximum charge per delay is selected for model input.

As a result, the deviating direction, horizontal distance, vertical distance, Euclidean distance, explosive type, burden, hole spacing, and maximum charge per delay are selected to predict the PPV.

3.3. Standardization of the Data. The values of all kinds of information vary greatly. The ratio of the largest to the smallest is more than 1000. It is necessary to standardize the input data to eliminate the influence of dimension and variation size. In this paper, the $Z$-score method, which is the most widely used standardized method at present, is used to standardize the input data of the model. The $Z$-score method is shown in equation (1). After establishment of the standardized model, all the prediction sample data need to be standardized according to the same model of the training samples.

$$
x_{i j}^{*}=\frac{\left(x_{i j}-x_{j}\right)}{\sigma_{j}} .
$$

\section{Prediction and Control of the PPV}

4.1. Principal Component Analysis (PCA) of Input Information. The input data of the ANN model contain eight kinds of variables according to Section 3.1. The input data contain considerable repetitive and redundant information, and an obvious correlation is found between each factor and PPV. For example, the layout characteristics of PPVs with different horizontal distances and Euclidean distances are similar. The reason for this phenomenon is that the horizontal distance has a high correlation with the Euclidean distance in the Ashele copper mine. The correlation coefficient between them is 0.930 . In other words, some information overlap exists. There are no apparent direct connections between the blasting parameters, such as the hole spacing and burden. If the explosive consumption is constant, the hole spacing is restricted by the burden. The hole spacing and burden are not completely independent. The existence of this repetitive and redundant information directly affects the prediction accuracy of PPV, which requires some data processing before use for the ANN model. The method of PCA extracts the key information to establish new variables that are unrelated to each other. At the same time, it deletes redundant information while making the best effort to preserve the original information. In this paper, PCA is used to eliminate the influence of the repetitive and redundant information included in the data of eight factors after standardization.

The error contribution of PCA is shown in Figure 5. The larger the variance percentage is, the greater the contribution of the principal component to the output. The variance contributions of the first four principal components are $39.20 \%, 31.75 \%, 14.43 \%$, and $7.78 \%$. The cumulative variance contribution of the first four principal components accounts for $93.16 \%$. In this paper, the first four principal components are selected to predict PPV. The eigenvalues of the four principal components are $3.136,2.540,1.154$, and 0.622 .

\subsection{Establishment of the PPV Prediction Model.} Generally, the training error decreases rapidly in the training process, while the testing error sometimes decreases with relatively narrow fluctuations. Referring to the early stopping method, the maximum number of learning sessions was 200,000 . Then, the best number of learning steps was determined to reduce the overfitting problem according to the error of learning and testing. In addition, a simple structure of the neural network model is helpful to avoid overfitting problems. Consequently, a topology with one hidden layer was used. The hidden layer had a node search range of 5-15. The neural network model had four inputs and one output. The population size was 200 , the total number of generations was 30 , the code string length was 15 , the crossover probability was 0.8 , the mutation probability was 0.2 , the inversion operator was 0.3 , and the initial weight was from -0.5 to 0.5 . The individual error and test target error were controlled at $10^{-6}$ and $10^{-5}$, respectively. The inertia coefficient was set to 0.5 , and the generation with a stable fitness 


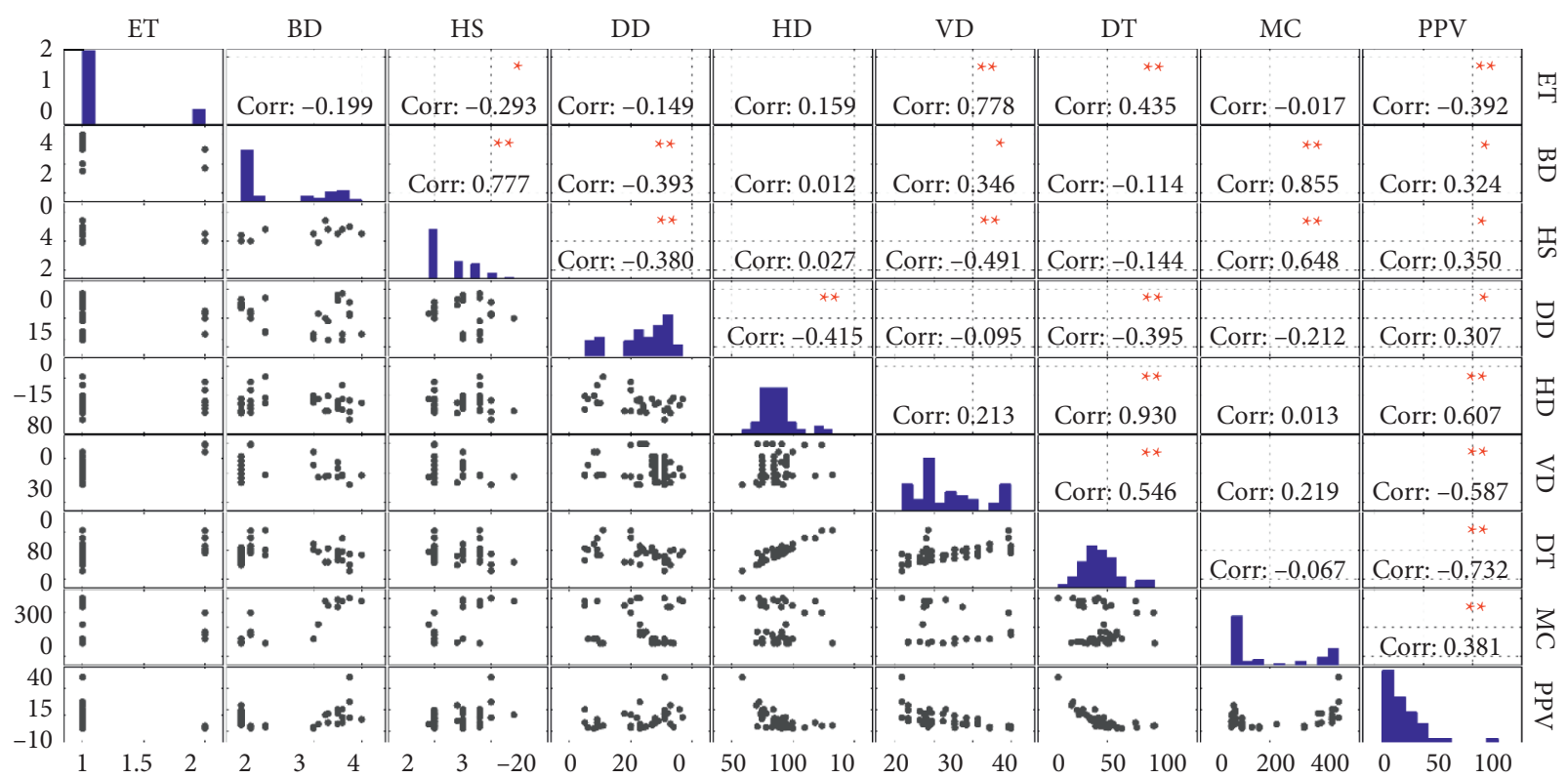

FIgURE 4: Scatterplot matrix of the PPV database with correlation. $*$ indicates that the correlation is significant at the 0.05 level, and $* *$ indicates that the correlation is significant at the 0.01 level.

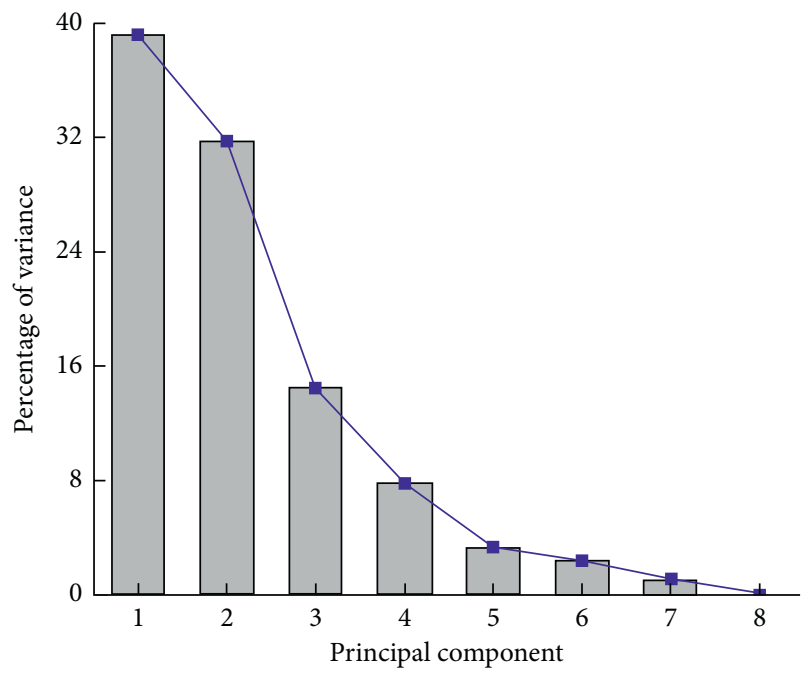

Figure 5: Variance contribution of each principal component.

value was set to 5 . In this paper, the mean squared error of the outputs from the measured results was chosen to calculate the fitness value.

The above parameter setting was used for the nine generations of genetic evolution. The best fitness value for each generation is shown in Figure 6. The optimal fitness value of the initial population was 1.711 . The best fitness value of the first-generation population was 1.700 , indicating a significant decrease. Then, a slight decrease in the best fitness value was found in the second generation. There was no significant change in the third and fourth generations, indicating that in the current genetic operation process, no chromosomes were better than those of the second generation. Afterwards, the best fitness value of the fifth generation was 1.207, showing a significant decrease and

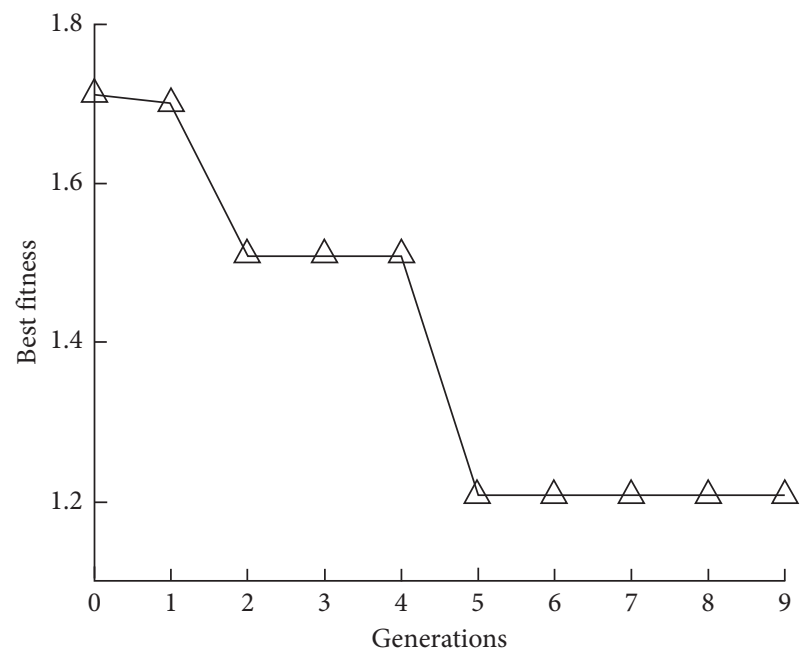

Figure 6: Best fitness values of different generations.

indicating that during the evolution from the fourth generation to the fifth generation, a better chromosome was generated, so the best fitness value was greatly reduced. Until the ninth generation, none of the individuals were better than those of the fifth generation. At this point, the generation with the stable best fitness value was set to 5 , and the genetic evolution was terminated. In this case, the best fitness values for the second to fourth generations were the same, as were the corresponding weights and topological parameters of the neural network. Therefore, the best fitness value and the corresponding weights and topological parameters of the neural network of any generation from the fifth generation to the ninth generation can be applied to the PPV neural network. The fitness values of each individual in the ninth generation and their corresponding topological parameters in the neural network are shown in Figure 7. 


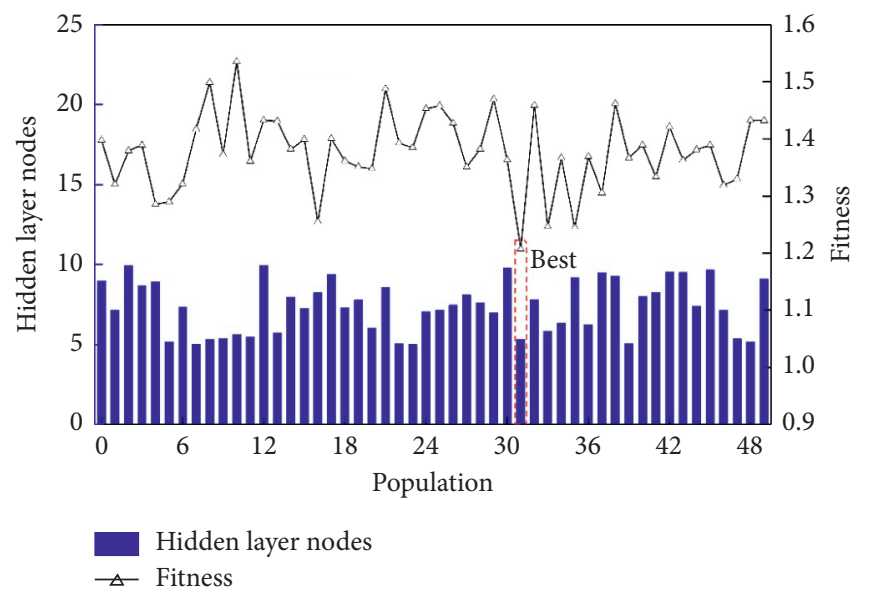

FiguRE 7: Hidden layer nodes of different generations.

There were 50 individuals in the population. The best fitness value of the ninth generation corresponded to individual no. 31 , and the topological parameter of its neural network was 5. Finally, the number of nodes in the hidden layer of the neural network was 5. Based on this, a PPV prediction model with a 4-5-1 topology was established. This model had one input layer, one hidden layer, and one output layer. The input layer contained four inputs, the hidden layer contained 5 nodes, and the output layer contained one output. In addition, there were 31 initial weights. The frame diagram of the three-layer neural network is shown in Figure 8.

The outputs of this model during learning and testing are shown in Figure 9. During learning and testing, the output values of the GA-ANN model were very close to the measured values. The errors of all learning and testing cases were within an acceptable range.

\subsection{Blasting Vibration Control for Crushing Cave Protection.} There is a crushing cave near the blasting stope at the $1012 \mathrm{~m}$ mining depth of the Ashele copper mine, which is shown in Figure 10. Two blasting vibration monitoring points were arranged near the stope to collect the PPV generated by the blasts. The blasting parameters and field conditions are shown in Table 1 . The PPV values at points 1 and 2 induced by large-hole blast were $116.1 \mathrm{~mm} / \mathrm{s}$ and $166.2 \mathrm{~mm} / \mathrm{s}$ according to the PPV prediction model and blasting design, respectively. The PPV at monitoring point 2 caused by a large-hole blast is close to the minimum safety value recommended in the blasting safety regulation. The blasting parameters were adjusted to control the blasting vibration to avoid damage to the crushing cave caused by blasts near the stope. The burden and hole spacing were changed to $3.5 \mathrm{~m}$ and $2.5 \mathrm{~m}$ from $3.3 \mathrm{~m}$ and $3.4 \mathrm{~m}$, respectively. Moreover, the maximum charge per delay was reduced from $400 \mathrm{~kg}$ to $300 \mathrm{~kg}$, while the delay number increased from 10 to 13 . The corresponding delay times were $0,50 \mathrm{~ms}, 110 \mathrm{~ms}, 150 \mathrm{~ms}$, $200 \mathrm{~ms}, 250 \mathrm{~ms}, 310 \mathrm{~ms}, 380 \mathrm{~ms}, 460 \mathrm{~ms}, 550 \mathrm{~ms}, 650 \mathrm{~ms}$, $760 \mathrm{~ms}$, and $880 \mathrm{~ms}$. After the adjustment of the blasting parameters, the predicted PPVs at monitoring points 1 and 2 were $55.60 \mathrm{~mm} / \mathrm{s}$ and $87.80 \mathrm{~mm} / \mathrm{s}$, respectively. Finally, the blast of the near stope did not cause apparent damage to the crushing cave. The measured PPVs at points 1 and 2 caused by the large-hole blast and medium-deep hole blast were $56.28 \mathrm{~mm} / \mathrm{s}$ and $96.99 \mathrm{~mm} / \mathrm{s}$, respectively.

\section{Discussion}

According to previous studies, the PPV is influenced by many factors, including environmental parameters such as the stress and rock mass quality and blasting parameters such as the maximum charge per delay, total charge, Euclidean distance, and number of delays. Most previous studies have considered the maximum charge per delay and the Euclidean distance to be the two most important parameters affecting the PPV. For example, empirical PPV predictors, such as those of the United States Bureau of Mines [32], Langefors and Kihlstrom [33], the Bureau of Indian Standards criteria [34], Ambraseys and Hendron [35], and the Central Mining Research Institute (CMRI) predictors [36], consider only the influences of the maximum charge per delay and the Euclidean distance on the PPV. These empirical formulas are shown in Table 2.

Using the learning and testing samples, the above five empirical models were used to establish the PPV prediction equations to predict the PPV values at different sites during the two blasts. The results predicted by the empirical equations were relatively poor (see Table 3 ). There is no significant difference between the PPV values predicted by the five empirical equations. This is because the same factors are taken into account by the five empirical equations with different expressions. The Ambraseys-Hendron equation was the best among these empirical equations for PPV prediction, and its prediction errors were approximately $13.36 \%$ and $87.81 \%$. As a result, the PPV values predicted by the GA-ANN algorithm were the closest to the measured values, and the prediction errors were less than $10 \%$, indicating that the prediction accuracy of the GA-ANN algorithm was the highest (see Figure 11). It is believed that the PPV prediction accuracy will be further improved with the accumulation of data. In general, the PPV should be predicted according to the actual factors rather than simply using empirical equations. 


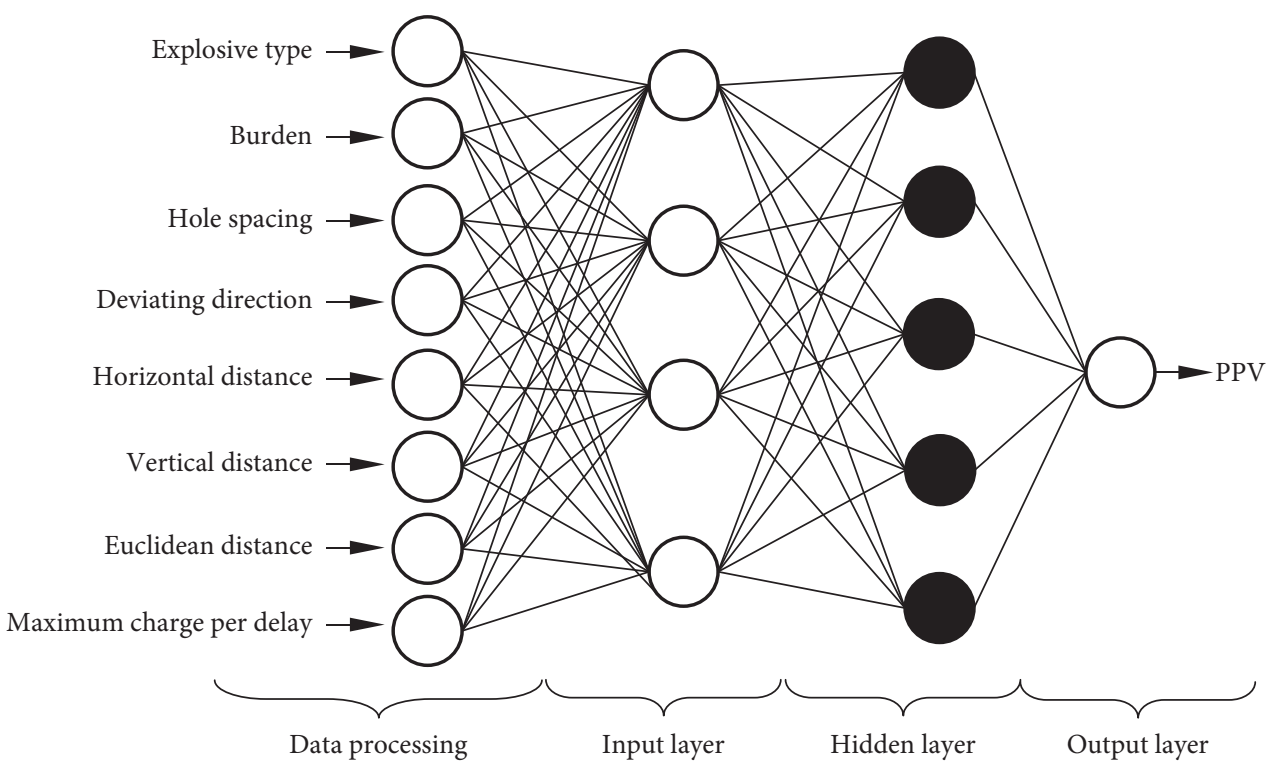

FIgURE 8: Frame diagram of the three-layer neural network.

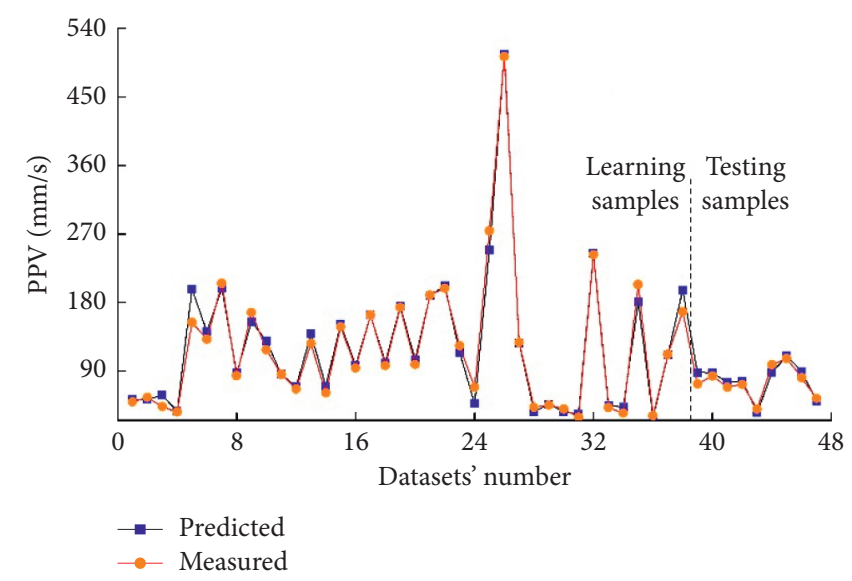

Figure 9: Relative errors of the training cases.

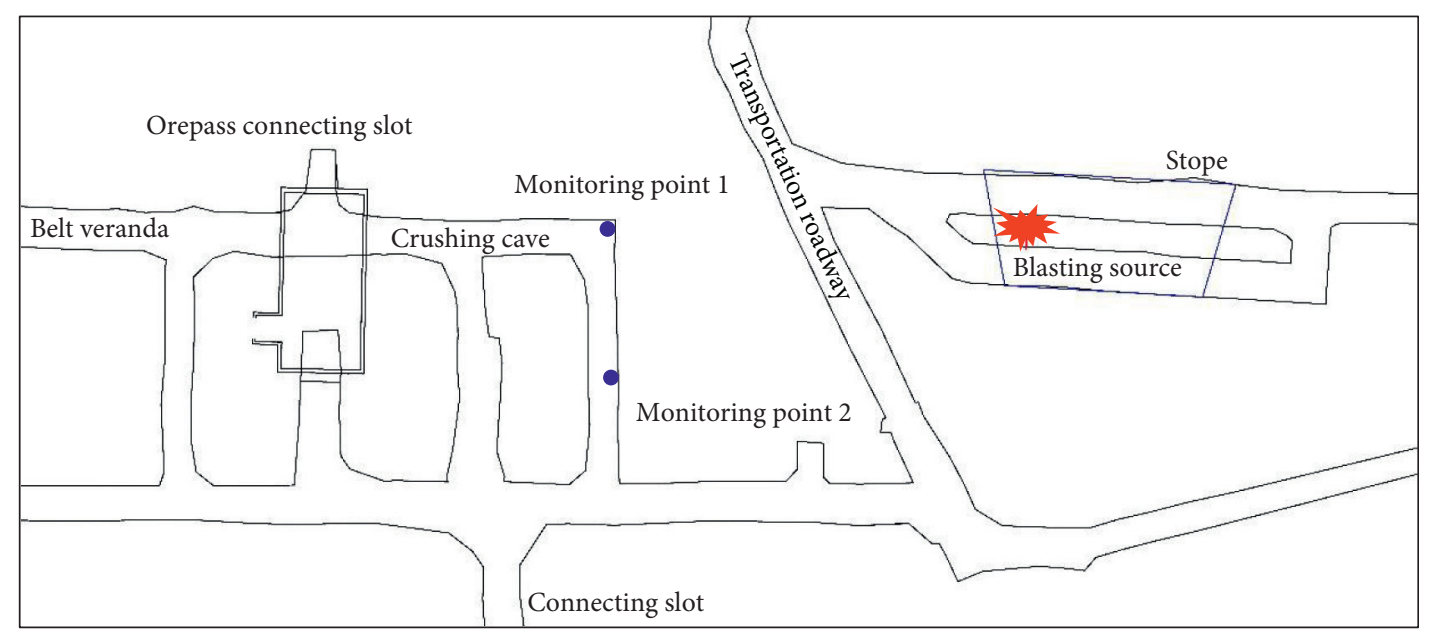

Figure 10: Location of the blasting source and crushing cave at a depth of $1012 \mathrm{~m}$ in the Ashele copper mine.

The traditional empirical formula considers only the blasting vibration Euclidean distance and the maximum charge per delay. In this paper, the Euclidean distances between the monitoring points and blasting source are close, while the maximum charges per delay are the same. Therefore, the predicted PPVs based on empirical formulas 
TABle 1: Parameters used to predict the PPV.

\begin{tabular}{lccccccc}
\hline $\begin{array}{l}\text { Explosive } \\
\text { type }\end{array}$ & $\begin{array}{c}\text { Burden } \\
(\mathrm{m})\end{array}$ & $\begin{array}{c}\text { Hole spacing } \\
(\mathrm{m})\end{array}$ & $\begin{array}{c}\text { Deviating } \\
\text { direction }\left(^{\circ}\right)\end{array}$ & $\begin{array}{c}\text { Horizontal } \\
\text { distance }(\mathrm{m})\end{array}$ & $\begin{array}{c}\text { Vertical } \\
\text { distance }(\mathrm{m})\end{array}$ & $\begin{array}{c}\text { Euclidean } \\
\text { distance }(\mathrm{m})\end{array}$ & $\begin{array}{c}\text { Maximum charge per } \\
\text { delay }(\mathrm{kg})\end{array}$ \\
\hline 1 & 3.3 & 3.4 & -2 & 46.02 & 17.5 & 49.24 & 400 \\
1 & 3.3 & 3.4 & -20 & 47.85 & 17.5 & 50.95 & 400 \\
1 & 3.5 & 2.5 & -2 & 46.02 & 17.5 & 49.24 & 300 \\
1 & 3.5 & 2.5 & -20 & 47.85 & 17.5 & 50.95 & 300 \\
\hline
\end{tabular}

TABLE 2: Different conventional predictors.

\begin{tabular}{lc}
\hline Name & Equation \\
\hline USBM & $v=K\left[R / \sqrt{Q_{\max }}\right]^{-B}$ \\
Langefors-Kihlstrom & $v=K\left[\sqrt{Q_{\max } / R^{2 / 3}}\right]^{B}$ \\
Ambraseys-Hendron & $v=K\left[R /\left(Q_{\max }\right)^{1 / 3}\right]^{-B}$ \\
Bureau of Indian Standards & $v=K\left[Q_{\max } / R^{2 / 3}\right]^{B}$ \\
CMRI predictor & $v=n+K\left[R / Q_{\max }\right]^{-1}$ \\
\hline
\end{tabular}

TABLE 3: Measured and predicted PPV values for different models.

\begin{tabular}{lccccc}
\hline \multirow{2}{*}{ Predictor } & \multicolumn{3}{c}{ Site constant } & \multicolumn{2}{c}{ PPV $(\mathrm{mm} / \mathrm{s})$} \\
& $K$ & $B$ & $n$ & 1 & 2 \\
\hline USBM & 345.38 & 1.02 & & 119.37 & 115.30 \\
Langefors-Kihlstrom & 13.31 & 1.54 & & 146.94 & 144.37 \\
Bureau of Indian & 13.31 & 0.77 & & 146.94 & 144.37 \\
Standards & 1100.46 & 1.15 & & 109.95 & 105.70 \\
Ambraseys-Hendron & 13.86 & & 46.29 & 130.71 & 127.88 \\
CMRI predictor & & & & 55.60 & 87.80 \\
GA-ANN & & & & 56.28 & 96.99 \\
Measured & & & & & \\
\hline
\end{tabular}

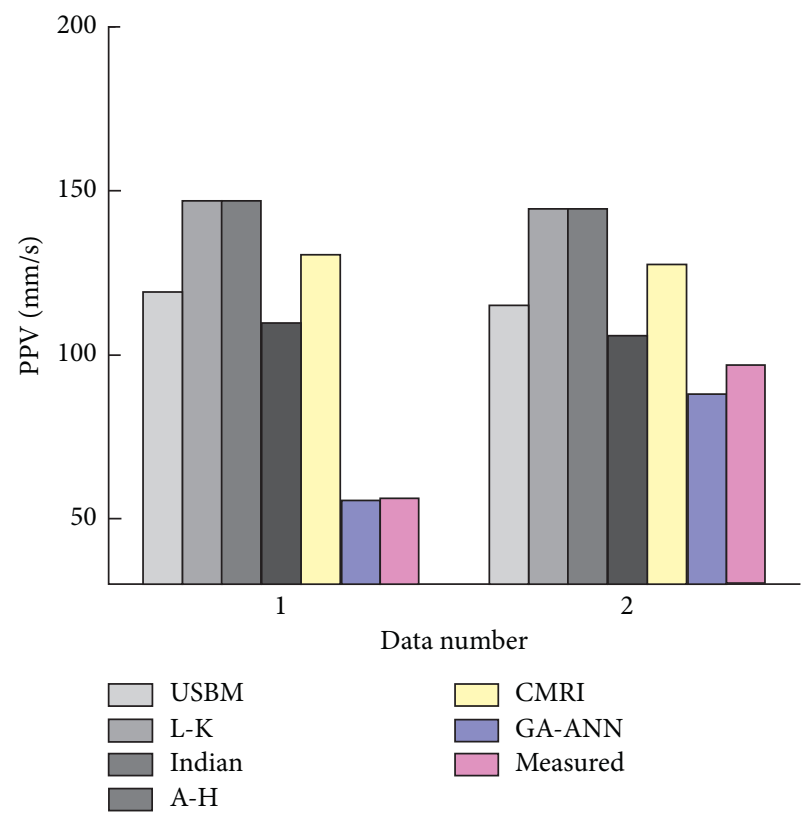

FIGURE 11: PPV values predicted by the GA-ANN model and the empirical models.

at two different monitoring points are very similar. In addition, the maximum charge per delay and Euclidean distance are found to significantly influence the PPV through correlation analysis. In fact, the influence of deviating direction, hole spacing, burden, and explosive type on the PPV cannot be ignored according to the data in this paper. Coupled with the advantages of neural networks in solving nonlinear problems, these reasons make the predicted PPV using the model in this paper more accurate than the empirical formulas. This can also be seen from the PPV prediction results of the two monitoring points. Compared with the traditional empirical formula, the predicted PPV values of the neural network at the two monitoring points with different deviating directions have marked differences.

\section{Conclusion}

This paper proposed a GA-ANN PPV prediction method. The input information of the PPV prediction method contains field conditions and blasting parameters, such as the deviating direction, horizontal distance, vertical distance, Euclidean distance, explosive type, burden, hole spacing, and maximum charge per delay. PCA was used to eliminate redundant and repetitive information of the field conditions and blasting parameters. The mean squared error of the outputs from the measured results was chosen as the fitting function to search for the best node number of the hidden layer. In this way, the GAANN PPV prediction model with a 4-5-1 topology was established for the Ashele copper mine. In the learning and testing data, the predicted results were in good agreement with the measured values. This model was used to predict and control the blasting vibration of a deep stope located at a $1012 \mathrm{~m}$ buried depth in the Ashele copper mine. Through the adjustment of blasting parameters, such as the maximum charge per delay, delay number, burden, and hole spacing, the blasting vibration was successfully reduced, and the crushing cave was protected from damage. By comparing the prediction results of the GA-ANN PPV prediction model with those of traditional empirical PPV prediction models, the superiority of the GA-ANN PPV prediction model was verified. Among the traditional PPV prediction methods, the Ambraseys-Hendron method was the best. The proposed GA-ANN PPV model can be used to guide the design of blasting parameters for field production.

\section{Data Availability}

The datasets used in the present study are available from the corresponding author upon reasonable request. 


\section{Conflicts of Interest}

The authors declare that they have no conflicts of interest regarding the publication of this paper.

\section{Acknowledgments}

This work was financially supported by project nos. 51704056 and 51974059 of the National Natural Science Foundation of China, project nos. 2017YFC0602904 and 2016YFC0801605 of the National Key Research and Development Plan of China, project no. 2017 M621152 of the Postdoctoral Science Foundation of China, and project nos. N160103006 and N180115010 of the Fundamental Research Funds for the Central University of China.

\section{References}

[1] E. Ghasemi, M. Ataei, and H. Hashemolhosseini, "Development of a fuzzy model for predicting ground vibration caused by rock blasting in surface mining," Journal of Vibration and Control, vol. 19, no. 5, pp. 755-770, 2013.

[2] N. Jiang, C.B. Zhou, X.D. Luo, and S.W. Lu, "Damage characteristics of surrounding rock subjected to VCR mining blasting shock," Shock and Vibration, vol. 2015, Article ID 373021, , 2015.

[3] M. Monjezi, M. Hasanipanah, and M. Khandelwal, "Evaluation and prediction of blast-induced ground vibration at Shur River Dam, Iran, by artificial neural network," Neural Computing and Applications, vol. 22, no. 7-8, pp. 1637-1643, 2013.

[4] M. Wang, X. Shi, and J. Zhou, "Charge design scheme optimization for ring blasting based on the developed Scaled Heelan model," International Journal of Rock Mechanics and Mining Sciences, vol. 110, pp. 199-209, 2018.

[5] Y.-h. Li, S.-d. Xu, and J.-p. Liu, "A new convergence monitoring system for tunnel or drift based on draw-wire displacement sensors," Tunnelling and Underground Space Technology, vol. 49, pp. 92-97, 2015.

[6] R.J. Durrheim, M.K.C. Roberts, A.T. Haile et al., "Factors influencing the severity of rock burst damage in South African gold mines," The Journal of South African Institute of Mining and Metallurgy, vol. 98, no. 2, pp. 53-57, 1998.

[7] Q. Jiang, X.-T. Feng, T.-B. Xiang, and G.-S. Su, "Rockburst characteristics and numerical simulation based on a new energy index: a case study of a tunnel at 2,500 m depth," Bulletin of Engineering Geology and the Environment, vol. 69, no. 3, pp. 381-388, 2010.

[8] G.A. Donald, T. Shahram, A. Keith, B. Patrice, and N.D. Stephen, "Ground vibrations produced by surface and near-surface explosions," Applied Acoustics, vol. 74, pp. 12791296, 2013.

[9] R. Guo, C.L. Pan, and R.C. Yu, Theory and Technology of Hard Rock Deposit with Rock Burst Tendency, Metallurgical Industry Press, Beijing, China, 2003.

[10] C. H. Dowding, "Suggested method for blast vibration monitoring," International Journal of Rock Mechanics and Mining Sciences \& Geomechanics Abstracts, vol. 29, no. 2, pp. 145-156, 1992.

[11] J. Zhou, X. Shi, and X. Li, "Utilizing gradient boosted machine for the prediction of damage to residential structures owing to blasting vibrations of open pit mining," Journal of Vibration and Control, vol. 22, no. 19, pp. 3986-3997, 2016.
[12] A. Kahriman, "Analysis of parameters of ground vibration produced from bench blasting at a limestone quarry," Soil Dynamics and Earthquake Engineering, vol. 24, no. 11, pp. 887-892, 2004.

[13] S. Xu, Y. Li, J. Liu, and F. Zhang, "Optimization of blasting parameters for an underground mine through prediction of blasting vibration," Journal of Vibration and Control, vol. 25, no. 9, pp. 1585-1595, 2019.

[14] S. Xu, C. Zhang, Z. Chen, Y. Li, and J. Liu, "Accurate identification of microseismic waveforms based on an improved neural network model," Journal of Applied Geophysics, vol. 190, Article ID 104343, 2021.

[15] T. N. Singh and V. Singh, "An intelligent approach to prediction and control ground vibration in mines," Geotechnical and Geological Engineering, vol. 23, no. 3, pp. 249-262, 2005.

[16] M. Khandelwal and T. N. Singh, "Prediction of blast induced ground vibrations and frequency in opencast mine: a neural network approach," Journal of Sound and Vibration, vol. 289, no. 4-5, pp. 711-725, 2006.

[17] M. Khandelwal and T. N. Singh, "Prediction of blast-induced ground vibration using artificial neural network," International Journal of Rock Mechanics and Mining Sciences, vol. 46, no. 7, pp. 1214-1222, 2009.

[18] M. Monjezi, M. Ghafurikalajahi, and A. Bahrami, "Prediction of blast-induced ground vibration using artificial neural networks," Tunnelling and Underground Space Technology, vol. 26, no. 1, pp. 46-50, 2011.

[19] H. Bakhshandeh Amnieh, A. Siamaki, and S. Soltani, "Design of blasting pattern in proportion to the peak particle velocity (ppv): artificial neural networks approach," Safety Science, vol. 50, no. 9, pp. 1913-1916, 2012.

[20] A. E. Álvarez-Vigil, C. González-Nicieza, F. López Gayarre, and M. I. Álvarez-Fernández, "Predicting blasting propagation velocity and vibration frequency using artificial neural networks," International Journal of Rock Mechanics and Mining Sciences, vol. 55, pp. 108-116, 2012.

[21] P. Jayawardana, D. P. Thambiratnam, N. Perera, and T. Chan, "Dual in-filled trenches for vibration mitigation and their predictions using artificial neural network," Soil Dynamics and Earthquake Engineering, vol. 122, pp. 107-115, 2019.

[22] A. K. Verma and T. N. Singh, "Intelligent systems for ground vibration measurement: a comparative study," Engineering with Computers, vol. 27, no. 3, pp. 225-233, 2011.

[23] M. T. Mohamed, "Performance of fuzzy logic and artificial neural network in prediction of ground and air vibrations," International Journal of Rock Mechanics and Mining Sciences, vol. 48, no. 5, pp. 845-851, 2011.

[24] D. J. Armaghani, M. Hajihassani, E. T. Mohamad, A. Marto, and S. A. Noorani, "Blasting-induced flyrock and ground vibration prediction through an expert artificial neural network based on particle swarm optimization," Arabian Journal of Geosciences, vol. 7, no. 12, pp. 5383-5396, 2014.

[25] M. Hajihassani, D. Jahed Armaghani, H. Sohaei, E. Tonnizam Mohamad, and A. Marto, "Prediction of airblast-overpressure induced by blasting using a hybrid artificial neural network and particle swarm optimization," Applied Acoustics, vol. 80, pp. 57-67, 2014.

[26] M. Hajihassani, D. Jahed Armaghani, A. Marto, and E. Tonnizam Mohamad, "Ground vibration prediction in quarry blasting through an artificial neural network optimized by imperialist competitive algorithm," Bulletin of Engineering Geology and the Environment, vol. 74, no. 3, pp. 873-886, 2015. 
[27] Y. Azimi, S. H. Khoshrou, and M. Osanloo, "Prediction of blast induced ground vibration (BIGV) of quarry mining using hybrid genetic algorithm optimized artificial neural network," Measurement, vol. 147, Article ID 106874, 2019.

[28] H. Nguyen, C. Drebenstedt, X.-N. Bui, and D. T. Bui, "Prediction of blast-induced ground vibration in an open-pit mine by a novel hybrid model based on clustering and artificial neural network," Natural Resources Research, vol. 29, no. 2, pp. 691-709, 2020.

[29] J. N. D. Gupta and R. S. Sexton, "Comparing backpropagation with a genetic algorithm for neural network training," Omega, vol. 27, no. 6, pp. 679-684, 1999.

[30] J.-p. Liu, R. Wang, G. Lei, Y.-t. Si, S.-d. Xu, and Y.-h. Li, "Studies of stress and displacement distribution and the evolution law during rock failure process based on acoustic emission and microseismic monitoring," International Journal of Rock Mechanics and Mining Sciences, vol. 132, Article ID 104384, 2020.

[31] J. Cohen, Statistical Power Analysis for the Behavioral Sciences, Lawrence Erlbaum Associates, Publishers, Hillsdale, NJ, USA, 2nd edition, 1988.

[32] W.I. Duvall and B. Petkof, Spherical propagation of explosion of generated strain pulses in rocks, pp. 21-22, US Bureau of Mines, Washington, DC, USA, 1958.

[33] U. Langefors and B. Kihlstrom, The Modern Technique of Rock Blasting, seconded, p. 405, Wiley Publisher, New York, NY, USA, 1973.

[34] Bureau of Indian Standards, Criteria for safety and design of structures subjected to underground blast, Bureau of Indian Standards, Manek Bhawan, New Delhi, India, 1973.

[35] N.R. Ambraseys and A.J. Hendron, "Dynamic behaviour of rock masses," Rock Mechanics in Engineering Practices, pp. 203-207, Wiley, London, UK, 1968.

[36] P. Roy, "Putting ground vibration predictors into practice," Colliery Guardian, vol. 241, pp. 63-67, 1993. 